\title{
The Accuracy And Influencing Factors of An Accelerometer-Based Portable Navigation System For Total Hip Arthroplasty In The Supine Position : A Prospective Multicenter Study Using 3D CT Measurement
}

Yukihide Minoda ( $\nabla$ yminoda@med.osaka-cu.ac.jp )

Osaka City University Graduate School of Medicine

Masayuki Ito

Aidu Chuo Hospital

Kentaro Iwakiri

Shiraniwa Hospital

Katsufumi Uchiyama

Kitasato University School of Medicine

Masashi Kawasaki

Aichi Kouseiren Konan Kosei Hospital

Akio Kanda

Juntendo Shizuoka Hospital

Tetsuya Jinno

Tokyo Medical and Dental University Medical Hospital

Ryo Sugama

Osaka City University Graduate School of Medicine

Daisuke Chiba

Tohoku University Hospital

Masahiro Hasegawa

Mie University Graduate School of Medicine

Takaaki Fujishiro

Takatsuki General Hospital

Research Article

Keywords: accuracy, accelerometer-based, supine, pelvic, Postoperative

Posted Date: September 22nd, 2021 
DOl: https://doi.org/10.21203/rs.3.rs-902612/v1

License: (c) (1) This work is licensed under a Creative Commons Attribution 4.0 International License. Read Full License 


\section{Abstract}

This multicenter prospective study aimed to evaluate the accuracy of a newly introduced accelerometerbased portable navigation system for total hip arthroplasty (THA) in the supine position. Three hundred twenty-four THAs using supine position in 9 hospitals were prospectively enrolled in the study. An accelerometer-based portable navigation system was used for cup fixation and the intraoperative navigation data (cup alignment, pelvic tilt, and pelvic rotation) were recorded. Postoperative cup alignment was measured on 3D-CT images. The accuracy (absolute difference in cup alignment between the intraoperative navigation record and postoperative 3D-CT measurements) was $3 \pm 3^{\circ}$ (mean \pm standard deviation) for cup inclination and $3 \pm 3^{\circ}$ for cup anteversion. The pelvis tilted anteriorly in 148 hips (46\%) and posteriorly in 162 hips (50\%), and did not tilt in 14 hips (4\%). The pelvis rotated toward the operating side in 179 hips (55\%), toward the contralateral side in 112 hips (35\%), and did not rotate in 33 hips $(10 \%)$. Multiple regression analysis showed that the patients' characteristics, pelvic tilt, and pelvic rotation did not affect the accuracy of the navigation system. The accuracy was $3 \pm 3^{\circ}$ and not affected by patient characteristics, surgical factors, or substantial pelvic movement during cup fixation.

\section{Introduction}

The orientation of the acetabular component during a total hip arthroplasty (THA) is of critical importance for realizing a good clinical outcome ${ }^{1-3}$. To improve the accuracy of cup alignment in THA, an accelerometer-based portable navigation system has recently been introduced for use in the supine position.

Accelerometer-based portable navigation systems, which provides lower cost and portability without a large computer and sensor, were introduced in 2016, with subsequent studies showing high levels of accuracy ${ }^{4-7}$. However, these reports were all single-institution studies and the procedures were performed by only a few surgeons. Therefore, the results of these reports may not be applicable to surgeons in other institutions. To our knowledge, there has been no multicenter prospective study to assess the accuracy of the accelerometer-based portable navigation system. Although the information about the accuracy of an accelerometer-based portable as a surgical instrument is very important for orthopaedic surgeons to decide whether or not to use this navigation system, the accuracy of the accelerometer-based portable navigation system is still controversial.

We therefore hypothesized that an accelerometer-based portable navigation system would provide the high accuracy of cup alignment in a multicenter study. The purpose of this multicenter prospective study was to evaluate the accuracy of cup alignment when utilizing an accelerometer-based portable navigation system during THA.

\section{Methods}


The protocol for this prospective study was approved by the institutional review board at Osaka City University Graduate School of Medicine in July 2017. This study was performed in accordance with the Declaration of Helsinki. Altogether, 324 THA patients in nine hospitals were prospectively enrolled in the study, which ran from October 2017 to November 2019. The inclusion criteria were that the patients (1) had osteoarthritis, avascular necrosis, or rheumatoid arthritis of the hip, and (2) required THA. Exclusion criteria included the presence of active infection ( $<6$ weeks preoperatively), pyogenic hip arthritis, osteomyelitis, or severe neurological disease. Informed consent was obtained from all patients prior to enrollment in the study.

All the operations were performed with the patients in the supine position, with either the direct anterior approach or the muscle-sparing modified Watson Jones anterolateral approach being used. All patients received the same prosthesis (G7; Zimmer Biomet, Warsaw, IN, USA). An accelerometer-based portable navigation system (HipAlign 2; OrthAlign; Aliso Viejo, CA, USA) was used in all cases. The reference sensor of the navigation system was set on the ipsilateral iliac crest using two pins. Registration needed three anatomical landmarks: both anterior superior iliac spines (ASISs) and the pubic tubercle. Pelvic flexion/extension was automatically detected by an accelerometer which was built into the navigation system. We recorded the preparation time for the navigation system, which was the time required for the fixation of the pins in the iliac crest and the registration of the anatomical landmarks. The navigation system indicated the cup alignment (inclination and anteversion). The aimed cup alignment was decided by the surgeons. The navigation system also indicated the pelvic tilt and pelvic rotation during the cup fixation. When the pelvis tilted anteriorly, the pelvic tilt was expressed as a positive value; when the pelvis tilted posteriorly, the pelvic tilt was expressed as a negative value. When the pelvis tilted toward the surgical side, the pelvic rotation was expressed as a positive value; when the pelvis tilted toward the contralateral side, the pelvic rotation was expressed as a negative value. Cup alignment, pelvic tilt, and pelvic rotation were expressed as integers.

A computed tomography (CT) scan of the pelvis was performed 2 weeks post-operatively. Postoperative cup alignment was measured on the 3D-CT images using computer software (ZedHip, LEXI, Tokyo, Japan). Cup alignment (inclination and anteversion) was calculated as an integer in radiographic terms ${ }^{8}$ by an experienced surgeon who had not participated in the THAs and was blinded to the patient information and the data indicated by the navigation system.

The intra-observer reliability of measurements for postoperative cup alignment was determined using intraclass correlation coefficients (ICCs) based on measurements of 20 patients, measured twice after an interval of $>4$ weeks. The ICCs were interpreted as follows: 0 to 0.40 , poor; 0.41 to 0.60 , moderate; 0.61 to 0.80 , good; and 0.81 to 1.00 , excellent ${ }^{9}$.

The primary aims of this study were 1) to determine the accuracy of the portable navigation system, which was defined as the absolute difference in cup alignment between the intraoperative record by the navigation system and the postoperative 3D measurement by computer software, and 2) to determine any outliers in the accuracy of the cup alignment, which were defined as any cases in which the absolute 
difference in accuracy measurements was $>5^{\circ}$ or $>10^{\circ}$. Secondary aims included determining pelvic tilt and pelvic rotation during the cup fixation, and factors affecting cup alignment.

\section{Statistical Analysis}

The cup alignments between the navigation system and the postoperative 3D-CT measurements were compared using paired t-tests, while pelvic tilt (anteriorly or posteriorly) and pelvic rotation (surgical side or contralateral side) were compared with Fisher's exact test. Single regression and stepwise multiple regression analyses were also conducted to determine the predictors of the dependent variable, namely the accuracy of the portable navigation system (inclination or anteversion). The independent variables were sex, age, height, body weight, body mass index, initial diagnosis, Crowe classification ${ }^{10}$, laterality, approach, preparation time for the navigation system, cup size, number of screws, pelvic tilt and pelvic rotation during cup fixation, and the institution. Bell Curve for Microsoft Excel (version 3.20; Social Survey Research Information) was used for the statistical analysis, and statistical significance was set at 0.05 .

\section{Results}

There was excellent reliability for measurements of cup inclination $(0.988 ; 95 \%$ confidence interval [Cl], 0.971 to 0.985$)$ and cup anteversion $(0.992 ; 95 \% \mathrm{Cl}, 0.979$ to 0.989$)$. There were no intraoperative complications resulting from using the accelerometer-based portable navigation system. The patients' demographic data are shown in Table 1.

\section{Primary Outcome}

The accuracy of the portable navigation system is shown in Table 2. The difference in accuracy between inclination and anteversion was not found to be statistically significant $(p=0.20)$. A histogram of the difference in cup alignment between the intraoperative records shown by the navigation system and the postoperative 3D-CT measurements are shown in Figure 1A and B. Outliers with $>5^{\circ}$ difference in accuracy were found in 54 hips $(17 \%)$ for inclination and 55 hips $(17 \%)$ for anteversion. Outliers with $>10^{\circ}$ difference in accuracy were found in 4 hips (1\%) for inclination and 6 hips (2\%) for anteversion.

\section{Secondary Outcome}

The intraoperative cup alignments indicated by the navigation system and the postoperative cup alignments using the 3D-CT measurement are shown in Table 3. The difference in measurements between the intraoperative and postoperative cup alignments was not statistically significant.

The degree of pelvic tilt and rotation during cup fixation is shown in Table 4, while the distribution of the pelvic tilt and rotation during cup fixation is shown in Figure $2 \mathrm{~A}$ and $\mathrm{B}$. The pelvis tilted anteriorly in 148 hips (46\%), posteriorly in 162 hips (50\%), and did not tilt in 14 hips (4\%). The pelvis rotated toward the operation side in 179 hips (55\%), toward the contralateral side in 112 hips (35\%), and did not rotate in 33 hips $(10 \%)$. There was no correlation found between pelvic tilt and pelvic rotation $(p=0.611)$. 
Single regression analysis showed that the age of the patient at the time of the operation correlated with the accuracy of cup anteversion ( $<<0.01$; Table 5). Additionally, stepwise multiple regression analysis showed that the patient's age at the time of the operation was identified as a predictor for the accuracy of cup anteversion $(R=0.176, p<0.01)$. However, the correlation coefficient was less than 0.2 , meaning that there was almost no correlation between the accuracy of cup anteversion and the patient's age at the time of the operation. None of the factors correlated with the accuracy of cup inclination.

\section{Discussion}

To the best of our knowledge, this is the first multicenter prospective study to evaluate the accuracy of an accelerometer-based portable navigation system for cup alignment during THA with the patient in the supine position. Our study showed that the accuracy was $3 \pm 3^{\circ}$ for cup inclination and $3 \pm 3^{\circ}$ for cup anteversion. Additionally, our study also showed that the patients' characteristics (sex, height, body weight, body mass index, diagnosis, Crowe classification, laterality, intraoperative pelvic tilt, intraoperative pelvic rotation, and cup size) and the surgical factors (approach, preparation time, number of screws, and institution) did not influence the accuracy of cup alignment. The accuracy of cup anteversion correlated with the patient's age at the time of the operation. However, the correlation coefficient was less than 0.2 $(R=0.176)$, meaning that the accuracy of cup anteversion and the patient's age at the time of the operation had almost no correlation. This study suggests that the accelerometer-based portable navigation system provides the same level of accuracy of cup alignment regardless of patient characteristics or surgical factors.

A previous systematic review showed that optical navigation systems were more accurate than freehand placement of the acetabular cup for both anteversion and inclination ${ }^{11}$. The accuracy of this multicenter study using an accelerometer-based portable navigation system was equivalent to the previous report using the CT-based optical navigation system in supine position, which requires preoperative CT, preoperative planning, complicated registration, a large sensor as well as a console outside the operative field, and additional personnel to operate the console ${ }^{12}$. The accuracy of this study was also equivalent to previous studies that reported on the use of image-free optical navigation systems in supine position for inclination but was better for anteversion ${ }^{12,13}$. The image-free navigation system needs to digitize both ASISs and the pubic tubercle for registration. However, an accelerometer-based portable navigation system only needs to digitize the ASISs. Digitizing the pubic tubercle does not affect cup alignment because pelvic tilt was automatically calculated by the built-in accelerometer. A previous report using a 3D pelvic model showed that the effect of potential digitization error at the pubic tubercle on the accuracy of cup anteversion is much larger than that at the ASISs ${ }^{14}$. In clinical situations, it is difficult to accurately digitize the pubic tubercle, particularly for obese patients. Tsukada et al. showed that errors in anteversion measurements when using an image-free navigation system were significantly more frequent in obese patients $\left(5 \pm 3^{\circ}\right)$ than in non-obese patients $\left(3 \pm 3^{\circ} ; p=0.01\right)^{15}$. Therefore, the accuracy of cup 
anteversion using an accelerometer-based portable navigation system that does not need to digitize the pubic tubercle is theoretically superior to the accuracy of an image-free optical navigation system which does need to digitize the pubic tubercle.

This study also showed that the pelvis moved substantially during the cup fixation in the spinal position. However, the direction of tilt and rotation was not constant. The pelvis tilted anteriorly in $46 \%$ of the cases, posteriorly in $50 \%$ of the cases, and did not tilt in $4 \%$ of the cases. Additionally, the pelvis rotated toward the operation side in $55 \%$ of the cases, toward the contralateral side in $35 \%$ of the cases, and did not rotate in $10 \%$ of the cases. There was no correlation found between pelvic tilt and pelvic rotation. This study suggested that pelvic movement during cup fixation was unpredictable, which might affect cup alignment using conventional techniques ${ }^{16}$. However, this study showed that the accelerometer-based portable navigation system was not affected by intraoperative pelvic movement.

This study has several strengths. First, postoperative cup alignment was measured by an experienced surgeon who had not participated in the THA procedure and who was blinded to the cup alignment indicated in the navigation system during the operation. This ensured that there was no bias regarding the evaluation of the prosthetic alignment. Second, prosthetic alignment was measured with the use of $3 \mathrm{D}$ computer software, so the ICCs for the radiographic evaluations were high, meaning that the measurement method for prosthetic alignment of this study was reliable. Third, the present study was a multicenter prospective study. Unlike previous reports that were performed at single institutions ${ }^{4-7}$, the present study reproduced a scenario that was closer to a normal clinical setting.

However, this study does have several limitations. First, the clinical scores and longevity were not evaluated. However, the primary aim of this study was to determine the accuracy of the newly introduced accelerometer-based portable navigation system as a surgical instrument in a clinical setting. A further long-term study, such as a national registry study, is needed to verify the effects of an accelerometer-based portable navigation system on clinical results such as longevity or dislocation. Second, all the operations were performed in the supine position. For lateral positions, the accelerometerbased portable navigation system will require another system for registration. Therefore, the results of this study cannot be directly applied to THA performed in the lateral position. Third, this study did not have a control group. This was because the various surgeons who participated in this multicenter study had different philosophies and techniques regarding aiming for cup alignment. Some surgeons aimed to set the cup with $45^{\circ}$ of inclination and $20^{\circ}$ of anteversion, others set the cup with $40^{\circ}$ of inclination and $15^{\circ}$ of anteversion, while others aimed different cup alignments for each patient according to the combined anteversion technique ${ }^{17}$ or combined target zone ${ }^{18}$ in order to prevent impingement between the stem neck and cup. If the aimed cup alignment was identical in all cases, a comparison of cup alignment between this navigation system and other systems such as manual technique would be clinically relevant. However, for this multicenter study, a comparison of postoperative cup alignment between an accelerometer-based navigation system and other techniques would have little clinical relevance, because the aimed cup alignment was different for each patient. Therefore, we did not use a control group. 
This study proved the accuracy of the newly introduced accelerometer-based navigation system as a surgical instrument. If this navigation system has greater accuracy than the systems that surgeons are currently using and they are also satisfied with the accuracy of this portable navigation system, then they should consider switching to this navigation system. However, if a surgeon needs even greater accuracy for cup alignment, they should consider using other systems such as a surgical robot.

This prospective multicenter study showed that the accuracy of an accelerometer-based portable navigation system for THA in the supine position was $3 \pm 3^{\circ}$ for cup inclination and $3 \pm 3^{\circ}$ for cup anteversion, although the pelvis moved substantially during the cup fixation. The results of this study may help orthopedic surgeons decide whether to use an accelerometer-based portable navigation system for future THAs.

\section{References}

1: Biedermann R, Tonin A, Krismer M, Rachbauer F, Eibl G, Stöckl B. Reducing the risk of dislocation after total hip arthroplasty: the effect of orientation of the acetabular component. J Bone Joint Surg Br. 2005;87:762-769.

2: Jolles BM, Zangger P, Leyvraz PF. Factors predisposing to dislocation after primary total hip arthroplasty: a multivariate analysis. J Arthroplasty. 2002;17:282-288.

3: Kennedy JG, Rogers WB, Soffe KE, Sullivan RJ, Griffen DG, Sheehan LJ. Effect of acetabular component orientation on recurrent dislocation, pelvic osteolysis, polyethylene wear, and component migration. J Arthroplasty. 1998;13:530-534.

4: Takada R, Jinno T, Miyatake K, Hirao M, Yagishita K, Yoshii T, Okawa A. Supine versus lateral position for accurate positioning of acetabular cup in total hip arthroplasty using the modified Watson-Jones approach: A randomized single-blind controlled trial. Orthop Traumatol Surg Res. 2019;105:915-922

5: Hayashi S, Hashimoto S, Takayama K, Matsumoto T, Kamenaga T, Fujishiro T, Hiranaka T, Niikura T, Kuroda R. Evaluation of the accuracy of acetabular cup orientation using the accelerometer-based portable navigation system. J Orthop Sci. 2020;25:612-617.

6: Okamoto M, Kawasaki M, Okura T, Ochiai S, Yokoi H. Comparison of accuracy of cup position using portable navigation versus alignment guide in total hip arthroplasty in supine position. Hip Int. 2020 Mar 3:1120700020908788. doi: 10.1177/1120700020908788. Epub ahead of print.

7: Hasegawa M, Naito Y, Tone S, Wakabayashi H, Sudo A. Accuracy of acetabular cup insertion in an anterolateral supine approach using an accelerometer-based portable navigation system. J Artif Organs. 2020 Sep 2. doi: 10.1007/s10047-020-01206-8. Epub ahead of print.

8: Murray DW. The definition and measurement of acetabular orientation. J Bone Joint Surg Br. 1993;75B:228-232. 
9: Koo TK, Li MY. A guideline of selecting and reporting intraclass correlation coefficients for reliability research. J Chiropr Med. 2016;15:155-163.

10: Crowe JF, Mani VJ, Ranawat CS. Total hip replacement in congenital dislocation and dysplasia of the hip. J Bone Joint Surg Am. 1979;61:15-23.

11: Snijders T, van Gaalen SM, de Gast A. Precision and accuracy of imageless navigation versus freehand implantation of total hip arthroplasty: A systematic review and meta-analysis. Int J Med Robot. 2017;13. doi: $10.1002 /$ rcs.1843.

12: Kalteis T, Handel M, Bäthis H, Perlick L, Tingart M, Grifka J. Imageless navigation for insertion of the acetabular component in total hip arthroplasty: is it as accurate as CT-based navigation? J Bone Joint Surg Br. 2006;88:163-167.

13: Lass R, Kubista B, Olischar B, Frantal S, Windhager R, Giurea A. Total hip arthroplasty using imageless computer-assisted hip navigation: a prospective randomized study. J Arthroplasty. 2014;29:786-791.

14: Lee YS, Yoon TR. Error in acetabular socket alignment due to the thick anterior pelvic soft tissues. J Arthroplasty. 2008 Aug;23(5):699-706.

15: Tsukada S, Wakui M. Decreased accuracy of acetabular cup placement for imageless navigation in obese patients. J Orthop Sci. 2010;15:758-763.

16: Kishimura Y, Minoda Y, Mizokawa S, Sugama R, Ohta Y, Nakamura H. Cup alignment in total hip arthroplasty using the musclesparing modifed Watson-Jones approach-comparison betweenlateral and supine positions. Int Orthop. 2019;43:2477-83.

17: Dorr LD, Malik A, Dastane M, Wan Z. Combined anteversion technique for total hip arthroplasty. Clin Orthop Relat Res. 2009;467:119-127.

18: Widmer KH. The Impingement-free, Prosthesis-specific, and Anatomy-adjusted Combined Target Zone for Component Positioning in THA Depends on Design and Implantation Parameters of both Components. Clin Orthop Relat Res. 2020;478:1904-1918.

\section{Tables}

Table 1: Patient demographic data 


\begin{tabular}{|c|c|}
\hline Parameter & Data \\
\hline Sex (female : male) (n) & $270: 54$ \\
\hline Age at operation (years old) & $66 \pm 12$ \\
\hline Height (cm) & $155 \pm 8$ \\
\hline Body weight (kg) & $57 \pm 11$ \\
\hline Body mass index $\left(\mathrm{kg} / \mathrm{m}^{2}\right)$ & $24 \pm 4$ \\
\hline \multicolumn{2}{|l|}{ Diagnosis (n) } \\
\hline Osteoarthritis & 294 \\
\hline Avascular necrosis of femoral head & 24 \\
\hline Rheumatoid arthritis & 6 \\
\hline \multicolumn{2}{|l|}{ Crowe classification (n) } \\
\hline Type I & 311 \\
\hline Type II & 12 \\
\hline Type II & 1 \\
\hline Laterality (right : left) (n) & $183: 141$ \\
\hline Approach (DAA : ALS) (n) & $112: 212$ \\
\hline Preparation time for navigation system (minutes) & $3.9 \pm 2.0$ \\
\hline \multicolumn{2}{|l|}{ Cup size (n) } \\
\hline $46 \mathrm{~mm}$ & 28 \\
\hline $48 \mathrm{~mm}$ & 95 \\
\hline $50 \mathrm{~mm}$ & 122 \\
\hline $52 \mathrm{~mm}$ & 40 \\
\hline $54 \mathrm{~mm}$ & 24 \\
\hline $56 \mathrm{~mm}$ & 6 \\
\hline $58 \mathrm{~mm}$ & 5 \\
\hline $60 \mathrm{~mm}$ & 3 \\
\hline $62 \mathrm{~mm}$ & 1 \\
\hline \multicolumn{2}{|l|}{ Number of cup screws (n) } \\
\hline 0 & 103 \\
\hline
\end{tabular}




\begin{tabular}{|l|l|}
1 & 192 \\
2 & 9 \\
3 & 18 \\
4 & 2 \\
\hline
\end{tabular}

*Mean and standard deviation were provided. DAA = direct anterior approach, ALS = muscle-sparing modified Watson-Jones anterolateral approach

Table 2: Accuracy of the portable navigation system for cup alignment

\begin{tabular}{|ll|}
\hline Parameter & Mean and standard deviation \\
\hline Inclination $\left(^{\circ}\right)$ & $3 \pm 3$ \\
\hline Anteversion $\left(^{\circ}\right)$ & $3 \pm 3$ \\
\hline
\end{tabular}

Table 3: Intraoperative and postoperative cup alignment

\begin{tabular}{|lllc|}
\hline & $\begin{array}{l}\text { Intraoperative alignment indicated by the } \\
\text { portable navigation system }\end{array}$ & $\begin{array}{l}\text { Postoperative alignment using } \\
\text { 3D-CT measurement }\end{array}$ & $\begin{array}{l}\text { P- } \\
\text { value }\end{array}$ \\
\hline $\begin{array}{l}\text { Inclination } \\
\left(^{\circ}\right)\end{array}$ & $39 \pm 3$ & $39 \pm 4$ & 0.12 \\
\hline $\begin{array}{l}\text { Anteversion } \\
\left(^{\circ}\right)\end{array}$ & $17 \pm 3$ & $17 \pm 5$ & 0.99 \\
\hline
\end{tabular}

Mean and standard deviation was provided.

Table 4: Intraoperative pelvic tilt and rotation during cup fixation

\begin{tabular}{|ll|}
\hline Parameter & Mean and standard deviation \\
\hline Pelvic tilt $\left(^{\circ}\right)^{\text {a }}$ & $-1 \pm 8$ \\
\hline Pelvic rotation $\left(^{\circ}\right)^{\text {b }}$ & $1 \pm 5$ \\
\hline
\end{tabular}

a: When the pelvis tilted anteriorly, the value was expressed as a positive value.

b: When the pelvis tilted toward the surgical side, the value was expressed as a positive value.

Table 5: Results of the Single Regression Analysis 
Independent variables Dependent Variables

\begin{tabular}{lllll}
\hline & \multicolumn{2}{l}{ Inclination } & \multicolumn{2}{l}{ Anteversion } \\
\hline Sex & $\mathrm{R}$ & $\mathrm{p}$ value & $\mathrm{R}$ & $\mathrm{p}$ value \\
\hline Age at operation & 0.010 & 0.86 & 0.032 & 0.57 \\
\hline Height & 0.022 & 0.70 & 0.176 & $<0.01$ \\
\hline Body weight & 0.055 & 0.32 & 0.010 & 0.85 \\
\hline Body mass index & 0.063 & 0.26 & 0.023 & 0.68 \\
\hline Diagnosis & 0.044 & 0.43 & 0.018 & 0.75 \\
\hline Crowe classification & 0.078 & 0.16 & 0.050 & 0.37 \\
\hline Laterality & 0.013 & 0.82 & 0.088 & 0.11 \\
\hline Approach & 0084 & 0.13 & 0.001 & 0.98 \\
\hline Preparation time & 0.025 & 0.66 & 0.025 & 0.65 \\
\hline Cup size & 0.086 & 0.12 & 0.013 & 0.82 \\
\hline Number of screws & 0.096 & 0.09 & 0.044 & 0.43 \\
\hline Pelvic tilt during the cup fixation & 0.018 & 0.74 & 0.039 & 0.48 \\
\hline Pelvic rotation during the cup fixation & 0.051 & 0.36 & 0.090 & 0.11 \\
\hline Institution & 0.013 & 0.82 & 0.020 & 0.72 \\
\hline
\end{tabular}

Figures 


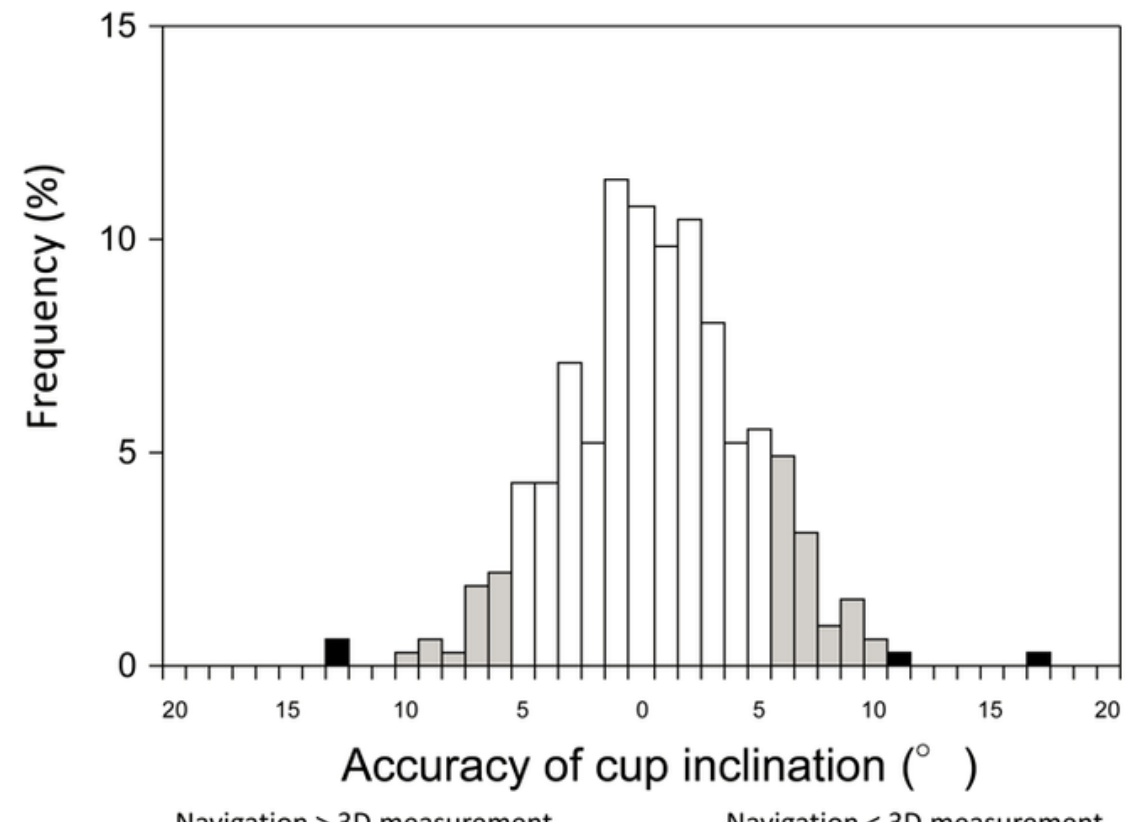

Navigation $>3 D$ measurement

Navigation $<3 D$ measurement

Figure 1A

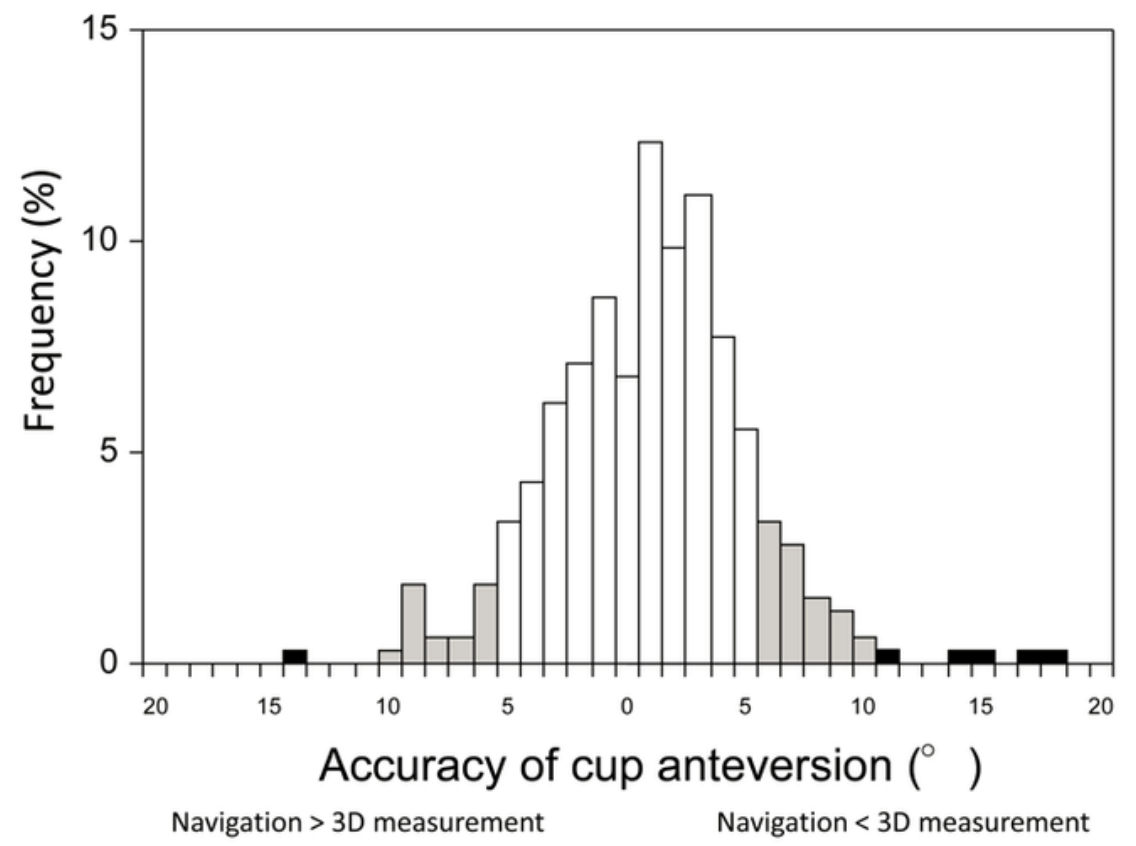

Figure 1B

\section{Figure 1}

A: Histogram showing the accuracy of cup inclination. Outliers with $>5^{\circ}$ difference in accuracy (gray and black bars) were found in 54 hips (17\%). Outliers with $>10^{\circ}$ difference in accuracy (black bars) were found in 4 hips (1\%). B: Histogram showing the accuracy of cup anteversion. Outliers with $>5^{\circ}$ difference in accuracy (gray and black bars) were found in 55 hips (17\%). Outliers with $>10^{\circ}$ difference in accuracy (black bars) were found in 6 hips (2\%). 


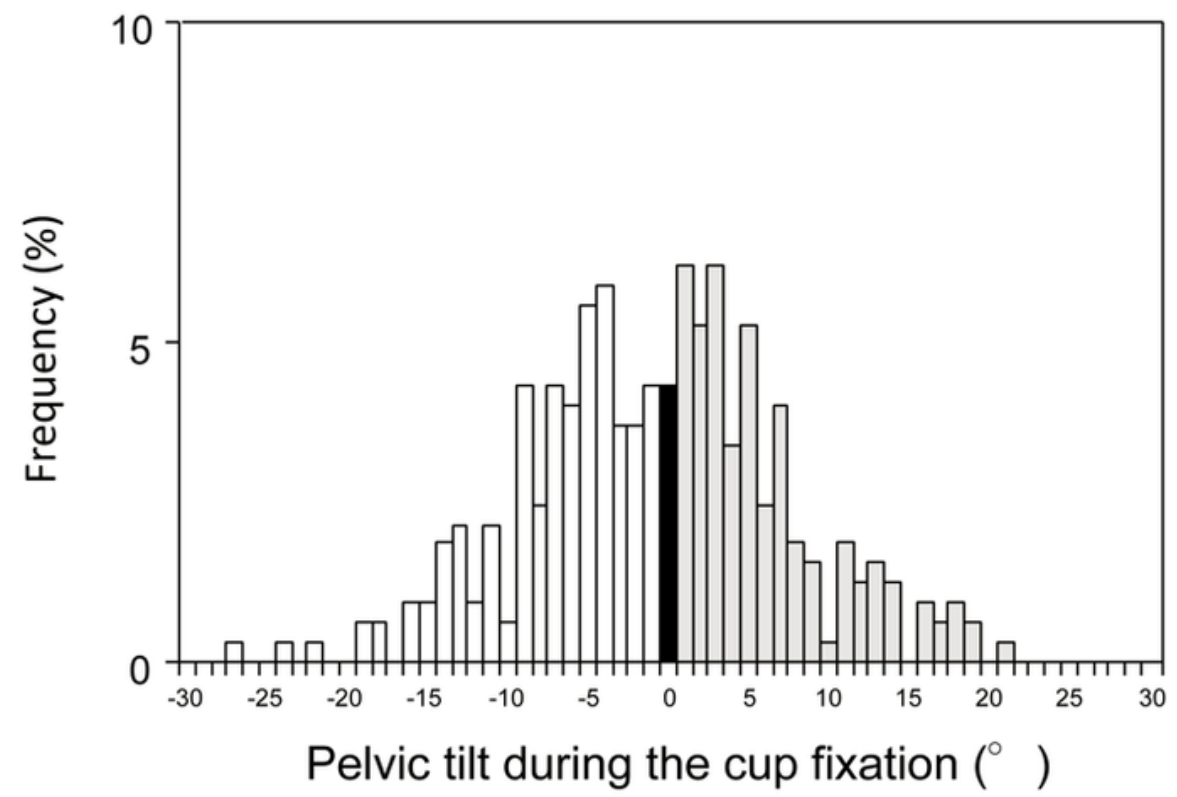

Figure 2A

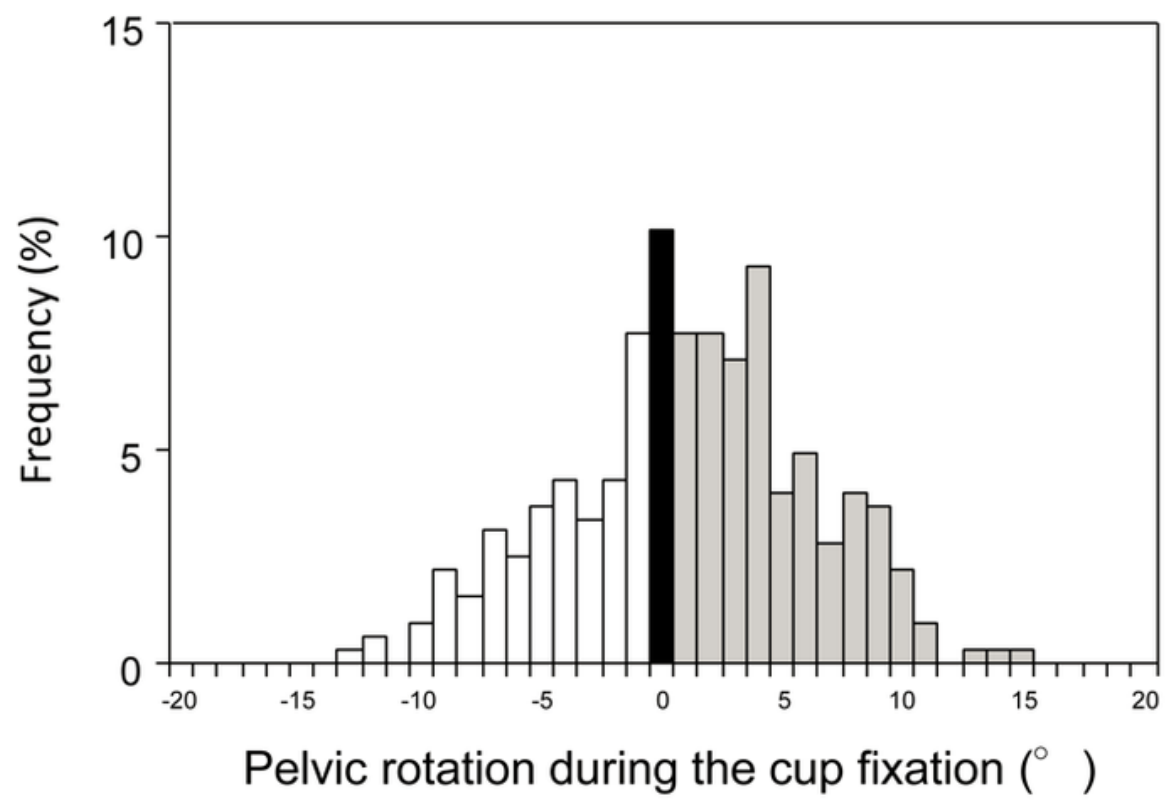

Figure 2B

Figure 2

A: Histogram showing the degree of pelvic tilt during cup fixation. When the pelvis tilted anteriorly, the pelvic tilt was expressed as a positive value (gray bars). When the pelvis tilted posteriorly, the pelvic tilt was expressed as a negative value (white bars). B: Histogram showing the degree of pelvic rotation during cup fixation. When the pelvis tilted toward the surgical side, the pelvic rotation was expressed as a 
positive value (gray bars). When the pelvis tilted toward the contralateral side, the pelvic rotation was expressed as a negative value (white bars). 